\title{
INFLUENCIA DE UN PROGRAMA DE SUPERVISIÓN EDUCATIVA EN EL CLIMA ORGANIZACIONAL DEL COLEGIO GARCÍA MORENO DEL CANTÓN EL GUABO - ECUADOR.
}

\section{INFLUENCE OF AN EDUCATIONAL SUPERVISION PROGRAM IN THE ORGANIZATIONAL CLIMATE OF GARCÍA MORENO SCHOOL IN EL GUABO CANTON - ECUADOR.}

\section{Autor: Richard Hugo Clavijo Flores}

E-mail de contacto: richard-valma@hotmail.com ORCID ID: https://orcid.org/0000-0002-0751-8588

Articulo recibido: 27 de Octubre del 2019

Articulo revisado: 2 de Noviembre del 2019

Articulo aprobado: 29 de Diciembre del 2019

Licenciado en Ciencias de la Educacion Especializacion Ciencias Sociales egresado de la Universidad Técnica de Machala (Ecuador) con 17 años de experiencia en la docencia. Posee una maestría en la Universidad Mayor San Marcos de Lima (Perú), mención Gestión de la Educación.

\section{Resumen}

La presente investigación titulada "Influencia de un programa de supervisión educativa en el clima organizacional del colegio García Moreno del cantón El Guabo - Ecuador 2019.", tiene como objetivo Determinar la influencia del Programa de supervisión educativa implementado en el Colegio García Moreno del cantón El Guabo, sobre el clima organizacional de la institución. $\mathrm{La}$ investigación es de tipo aplicada, de alcance explicativa, el diseño utilizado es experimental, de corte longitudinal. La muestra estuvo formada por 25 docentes tanto para el grupo control y 25 para el grupo experimental en el Colegio García Moreno del cantón El Guabo; para recolectar datos en cuanto a la variable clima organizacional se utilizó como técnica la encuesta y como instrumento un cuestionario el cual pasó por los procesos de validez y confiabilidad. El procesamiento de datos se realizó con el software SPSS (versión 23). Realizado el análisis descriptivo y después de interpretar los resultados del pre test y el del postest en el cual se obtuvo que el valor de $\rho=$ 0.000 ( $\mathrm{p}<0.05)$, con el cual se rechaza la hipótesis nula por lo tanto los resultados señalan. El clima organizacional en el colegio García Moreno del cantón El Guabo en Ecuador, mejora significativamente por influencia la implementación del programa de supervisión educativa.
Palabras claves: Supervisión, clima organizacional, institución, docentes.

\begin{abstract}
This research entitled "Influence of an educational supervision program in the organizational climate of Garcia Moreno School of the canton of Guabo - Ecuador 2019.", which aims to determine the influence of the educational supervision program implemented in the Garcia School Moreno del canton El Guabo, about the organizational climate of the institution. The research is of applied type, of explanatory scope, the design used is experimental, of longitudinal cut. The sample consisted of 25 teachers for both the control group and 25 for the experimental group at the Garcia Moreno School in the canton of Guabo; To collect data regarding the organizational climate variable, the survey was used as a technique and as a tool a questionnaire which went through the 'validity and reliability' processes. Data processing was performed with the SPSS software (version 23). Performed the descriptive analysis and after interpreting the results of the pretest and the posttest in which it was obtained that the value of $\rho=0.000(p<0.05)$, with which the null hypothesis is rejected, therefore the results indicate. The organizational climate at García Moreno School in the canton of Guabo in Ecuador, significantly improves the influence
\end{abstract}


of the implementation of the Educational Supervision Program.

\section{Keywords Supervision, organizational climate, institution, teachers.}

\section{Sumário}

Esta pesquisa intitulada "Influência de um programa de supervisão educacional no clima organizacional da Escola Garcia Moreno, no cantão de Guabo - Equador - 2019.", objetiva determinar a influência do programa de supervisão educacional implementado na Escola Garcia Moreno del cantón El Guabo, sobre o clima organizacional da instituição. A pesquisa é do tipo aplicada, de escopo explicativo, com delineamento experimental, de corte longitudinal. A amostra foi composta por 25 professores para o grupo controle e 25 para o grupo experimental da Escola Garcia Moreno, no cantão de Guabo; Para coletar dados referentes à variável clima organizacional, a pesquisa foi utilizada como técnica e como ferramenta, como questionário, que passou pelos processos de 'validade e confiabilidade'. O processamento dos dados foi realizado com o software SPSS (versão 23). Realizou a análise descritiva e após a interpretação dos resultados do pré-teste e pósteste em que foi obtido o valor de $\rho=0,000$ (p $<0,05)$, com o qual a hipótese nula é rejeitada, portanto, os resultados indicam. O clima organizacional da Escola García Moreno, no cantão de Guabo, no Equador, melhora significativamente a influência da implementação do Programa de Supervisão Educacional.

\section{Palavras-chave Supervisão, clima organizacional, instituição, professores.}

\section{Introducción}

La supervisión es un proceso cuya finalidad principal es mejorar el funcionamiento de las organizaciones humanas. En el caso específico de la educación, debe contribuir a lograr aprendizajes de mayor calidad, con la finalidad de elevar la calidad de la enseñanza. La realimentación del proceso educativo no sería completa sin adecuados mecanismos de supervisión.

El currículo podrá también afectarse positivamente por la supervisión, evaluarse, perfeccionarse, adaptarse en función de los resultados de la evaluación (supervisión) de la labor educativa. Así mismo será posible la implementación de actividades extracurriculares que complementen la formación de los estudiantes, en función de las características socioculturales y las necesidades de su comunidad.

El clima organizacional, entendido como aquellas percepciones de los profesionales sobre los comportamientos organizativos que afectan a su rendimiento en el trabajo; formales o informales, que influyen positiva o negativamente en el rendimiento del trabajo; es un aspecto de vital importancia en las entidades educativas, independientemente de su naturaleza o nivel. Se construye de modo permanente sobre la cultura de la organización. Las expectativas, la actitud y respuestas de los miembros de la organización ante cualquier estímulo en la labor cotidiana, están fuertemente condicionadas por el clima que en la organización sea dominante.

Al respecto, Alvarado (2002) afirma que "La supervisión constituye un método de investigación descriptiva sobre los diversos aspectos o variables del quehacer educativo, mediante el cual se recoge información, se la procesa, analiza y evalúa adecuadamente para ofrecer alternativas de solución pertinentes sobre los diversos problemas detectados" ( $p$. 28). Es menester que la entidad supervisora, logre que este proceso se constituya en una instancia para el reconocimiento de los méritos en la práctica pedagógica, que se administren reforzadores positivos; que se convierta en una 
oportunidad para acceder a la capacitación y asesoramiento permanente; además; que esté imbuido de los principios democráticos que deben guiar la conducción de una entidad educativa. De esta manera, la supervisión contribuirá al mejoramiento del clima organizacional.

En concordancia con Alvarado (2002), se puede sostener que mientras más rasgos democráticos tengan el proceso de supervisión, más probabilidades habrán de lograr que los resultados de la supervisión produzcan mayor motivación para la actividad docente, satisfacción laboral y como consecuencia, mayor eficiencia en la enseñanza. De otro lado, se tendrá menos rechazo, descontento, frustración y expresiones de agresividad. Las actitudes de solidaridad, ayuda mutua, se fortalecerán; dotando así a la institución, de mayores recursos para la resolución de los problemas propios de la acción educativa.

La supervisión educativa tiene significativa importancia en la organización y funcionamiento del sistema educativo debido a que impulsa la innovación, el desarrollo, la creatividad, motivación, clima organizacional y demás aspectos necesarios que deben estar presentes en una institución educativa. El tratamiento democrático de la supervisión viene siendo una importante coincidencia en la literatura especializada. La calidad de la educación depende de este factor de modo gravitante. Aunque no se puede establecer una relación mecánica de causa - efecto entre supervisión democrática y calidad de la educación, es posible afirmar se constituye en una relación determinante, tal como lo sostiene García (2015: 131), "la calidad de la supervisión escolar se encamina a orientar, ayudar y asesorar al docente a desarrollar lazos morales con los miembros de la comunidad donde se desenvuelve".

La supervisión educativa debe responder a las demandas del clima organizacional de una institución educativa con la finalidad de mejorar la comunicación, estar acorde con los adelantos de la ciencia y tecnología, mantener motivados a quienes forman parte de la institución y, sobre todo trabajar en armonía con el talento humano.

En este contexto se presenta la investigación titulada "Influencia de un programa de supervisión educativa en el clima organizacional del colegio García Moreno del cantón El Guabo - Ecuador - 2019.” La misma que permite realizar un análisis de la forma como se lleva a cabo la supervisión educativa en la institución y plantear alternativas de solución a la problemática. Por ello, se plantea como objetivo general: determinar la influencia del programa de supervisión educativa implementado en el Colegio García Moreno del cantón El Guabo, sobre el clima organizacional de la institución.

\section{Desarrollo}

\section{Supervisión educativa}

La supervisión educativa tradicionalmente ha estado orientada a la verificación de las condiciones del aprendizaje, con el fin de mejorar. Alvarado (2001, p. 22), propone una supervisión orientada al control de calidad, es decir centrada en el diseño (programación), proceso (Proceso de enseñanza-aprendizaje) y en los resultados (Evaluación).

Esta supervisión puede presentar una serie de ventajas: prevención de deficiencias, uniformidad de los procesos y productos; incremento de la productividad; mayor aceptación de parte de la sociedad; flujo de 
información retro alimentadora; mejoramiento continuo e innovación, reducción de costos.

Hay diversas concepciones del término supervisión. Según Alvarado (2001pp. 26-28) considera que es un servicio, una función, un método, una disciplina, una actividad y un sistema. Inicialmente la supervisión educativa se instituye como un servicio indispensable para ayudar a orientar el trabajo de los diversos agentes de la educación. Este servicio no desconoce la capacidad del profesor, sino que a partir de su reconocimiento le posibilita su auto superación. En este sentido, Alvarado (2001:26) sostiene que la supervisión debe ser una acción pedagógica democrática que ayude a los docentes lograr mejores niveles de desempeño y desarrollo personal.

También, se le concibe como una función de responsabilidad de los directivos, casi siempre de los subdirectores y jerárquicos, quienes deben cumplir esta función al interior de su propia entidad, sea de manera individual o en equipo. En este sentido, la supervisión puede entenderse como función, en la medida que la administración educativa, con el fin de dinamizar el sistema educativo, asigna a la supervisión como una función inherente a los cargos directivos y jerárquicos, en las instituciones educativas.

Así mismo, se considera como un método porque si se ejecuta técnicamente, requiere de un método de trabajo o de una secuencia metodológica adecuada. Es decir, la supervisión constituye un método de investigación descriptivo sobre diversos aspectos o variables del quehacer educativo, mediante el cual se recoge información, se la procesa, analiza y evalúa.

\section{Clima Organizacional}

El clima organizacional, según Martín (2001:213), son percepciones de los profesionales sobre los comportamientos organizativos que afectan su rendimiento en el trabajo. Velasco (2000:159), afirma que el clima organizacional es la personalidad de una organización. Para (Mujica, M. y Pérez, I. 2007:6) el clima organizacional es un factor importante que arbitra entre los factores del sistema organizacional y el comportamiento individual

Por su parte, Lepeley (2001:105) define el clima organizacional como "El ambiente social de la institución". Bustos (2001: 7), coincide con los autores anteriores y sostiene que el clima, es la cualidad o propiedad del ambiente organizacional, que perciben o experimentan los miembros de la organización, y que influyen en su comportamiento. Para que una persona pueda trabajar bien debe sentirse bien consigo mismo y con todo lo que gira alrededor de ella $\mathrm{y}$ entender el ambiente donde se desenvuelve todo el personal. Agrega, que un buen clima o un mal clima organizacional, tendrá consecuencias para la organización a nivel positivo y negativo, definidas por la percepción que los miembros tienen de la organización. Entre las consecuencias positivas, podemos nombrar las siguientes: logro, afiliación, poder, productividad, baja rotación, satisfacción, adaptación, innovación, etc. Entre las consecuencias negativas, podemos señalar: inadaptación, alta rotación, ausentismo, poca innovación, baja productividad, etc.

Según Quevedo (2006:21) es el grupo de características que describen a una organización que la hacen diferente de otras organizaciones; e influyen en la conducta de las personas en la organización. En el mismo sentido, Sandoval (2007: 2), lo define como: "El ambiente de 
trabajo percibido por los miembros de la organización y que incluye estructura, estilo de liderazgo, comunicación, motivación y recompensas, todo ello ejerce influencia directa en el comportamiento y desempeño de los individuos". (Salguero, 2017:2) coincide con los autores anteriores al considerar el clima "como las percepciones que los miembros de una organización tienen sobre ella, es el ambiente de trabajo propio de la organización, el mismo que ejerce influencia directa en la conducta y el comportamiento de sus trabajadores y directivos".

Consideramos entonces, que el Clima Organizacional es el ambiente social que perciben los miembros de una institución, el cual es determinante en la forma que toma una organización, en las decisiones que al interior de ella se ejecutan o en cómo se tornan las relaciones dentro y fuera de la organización.

Por lo expuesto, el clima organizacional tiene significativa importancia en una institución educativa porque de ello depende las relaciones comunicativas, el tipo de liderazgo, la motivación de todos sus miembros, entre otros aspectos.

\section{Material y Métodos}

Instrumentos de recolección de datos

En el estudio se hizo uso de la técnica del cuestionario, por la modalidad de estudio y el tiempo de aplicación, al respecto se utilizó el instrumento cuestionario.

El cuestionario: Es un instrumento de investigación. Este instrumento se utiliza, de un modo preferente, en el desarrollo de una investigación en el campo de las ciencias sociales científico y objetivo. Elaborar un cuestionario válido no es una cuestión fácil; implica controlar una serie de variables. Es un medio útil y eficaz para recoger información en un tiempo relativamente breve. (Hernández et al, 2010). En su construcción pueden considerarse preguntas cerradas, abiertas o mixtas.

\section{Diseño de estudio:}

Fue descriptivo correlacional y el diseño utilizado es no experimental, de corte transversal. Es cuasi experimental y que se recogen datos de muestras independientes y muestras relacionadas, mediante la aplicación de instrumentos de pre-test y post-test, con una escala de Likert bipolar, se pretende demostrar la existencia de relaciones significativas mediante la estadística no paramétrica Según (Berlanga \& Rubio, 2012), las pruebas no paramétricas engloban una serie de pruebas estadísticas que tienen como denominador común la ausencia de ciertos supuestos acerca de la ley de probabilidad que sigue la población de la que ha sido extraída la muestra. Por esta razón, es común referirse a ellas como pruebas de distribución libre.

\section{Resultados}

Resultados descriptivos del clima
organizacional

Tabla $N^{\circ} 1$ Antes de la aplicación del programa

\begin{tabular}{|l|c|c|c|c|}
\hline & \multicolumn{2}{|c|}{ Grupo control } & \multicolumn{2}{c|}{ Grupo experimental } \\
\hline & $\mathrm{f}$ & $\%$ & $\mathrm{f}$ & $\%$ \\
\hline Siempre & 0 & $0 \%$ & 0 & $0 \%$ \\
\hline Casi siempre & 1 & $4 \%$ & 0 & $0 \%$ \\
\hline A veces & 7 & $28 \%$ & 7 & $20 \%$ \\
\hline Casi nunca & 17 & $68 \%$ & 18 & $80 \%$ \\
\hline Nunca & 0 & $0 \%$ & 0 & $0 \%$ \\
\hline
\end{tabular}

Fuente: Base de datos

No obstante, lo anterior, su construcción, aplicación y tabulación poseen un alto grado 
Tabla $N^{\circ} 2$ Después de la aplicación del programa

\begin{tabular}{|l|c|c|c|c|}
\hline & \multicolumn{2}{|c|}{ Grupo control } & \multicolumn{2}{c|}{$\begin{array}{l}\text { Grupo } \\
\text { experimental }\end{array}$} \\
\hline & f & $\%$ & f & $\%$ \\
\hline Siempre & 0 & $0 \%$ & 0 & $0 \%$ \\
\hline Casi siempre & 0 & $0 \%$ & 19 & $84 \%$ \\
\hline A veces & 8 & $28 \%$ & 6 & $16 \%$ \\
\hline Casi nunca & 17 & $72 \%$ & 0 & $0 \%$ \\
\hline Nunca & 0 & $0 \%$ & 0 & $0 \%$ \\
\hline
\end{tabular}

Fuente: Base de datos

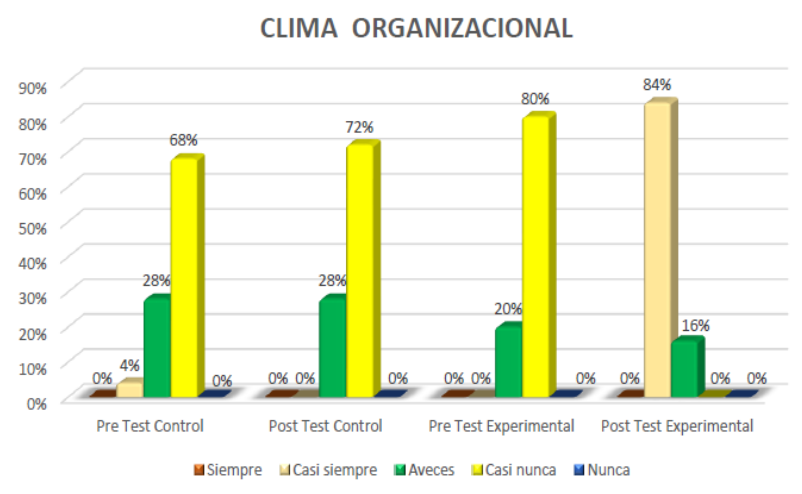

Figura 1. Niveles de la variable clima organizacional

En las tablas 1; 2 y en la figura 1 se observa que en el pre test con respecto al grupo control el $4 \%$ en el nivel casi siempre y el $28 \%$ a veces y el $68 \%$ casi nunca; mientras que en el grupo experimental el $20 \%$ consideró a veces, el $80 \%$ casi nunca. En el pos test se observa que en el grupo control, el $28 \%$ consideró a veces, y el $72 \%$ casi nunca, mientras en el grupo experimental el $84 \%$ consideró casi siempre y el $16 \%$ a veces; este resultado evidencia la mejora en el grupo experimental respecto al clima organizacional en el colegio García Moreno del cantón El Guabo después de realizarse la intervención del programa de supervisión educativa.

Resultados descriptivos de la apertura a los cambios tecnológicos.

Tabla 3 Antes de la aplicación del programa

\begin{tabular}{|l|c|c|c|c|}
\hline & \multicolumn{2}{|c|}{ Grupo control } & \multicolumn{2}{c|}{ Grupo experimental } \\
\hline & $\mathrm{f}$ & $\%$ & $\mathrm{f}$ & $\%$ \\
\hline Siempre & 0 & $0 \%$ & 0 & $0 \%$ \\
\hline Casi siempre & 1 & $4 \%$ & 1 & $4 \%$ \\
\hline A veces & 6 & $24 \%$ & 7 & $28 \%$ \\
\hline Casi nunca & 17 & $68 \%$ & 13 & $52 \%$ \\
\hline Nunca & 1 & $4 \%$ & 4 & $16 \%$ \\
\hline
\end{tabular}

Fuente: Base de datos
Tabla 4 Después de la aplicación del programa

\begin{tabular}{|l|c|c|c|c|}
\hline & \multicolumn{2}{|c|}{ Grupo control } & \multicolumn{2}{c|}{ Grupo experimental } \\
\hline & $\mathrm{f}$ & $\%$ & $\mathrm{f}$ & $\%$ \\
\hline Siempre & 0 & $0 \%$ & 2 & $8 \%$ \\
\hline Casi siempre & 1 & $4 \%$ & 17 & $68 \%$ \\
\hline A veces & 7 & $28 \%$ & 5 & $20 \%$ \\
\hline Casi nunca & 16 & $64 \%$ & 1 & $4 \%$ \\
\hline Nunca & 1 & $4 \%$ & 0 & $0 \%$ \\
\hline
\end{tabular}

Fuente: Base de datos

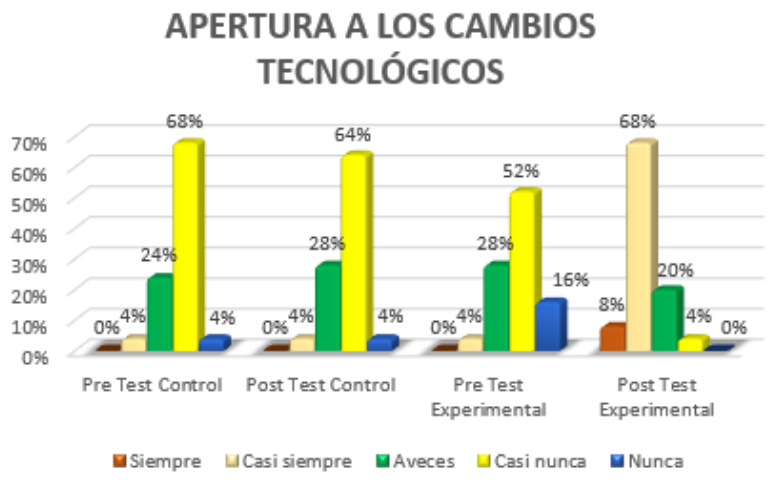

Figura 2. Niveles de la dimensión apertura a los cambios tecnológicos.

En las tablas 3; 4 y en la figura 2 se observa que en el pre test con respecto al grupo control el $4 \%$ consideró casi siempre y el $24 \%$ a veces y el $68 \%$ casi nunca y el $4 \%$ nunca; mientras que en el grupo experimental el $4 \%$ casi siempre, el $28 \%$ consideró a veces, el $52 \%$ casi nunca y el $16 \%$ nunca.

En el pos test se observa que en el grupo control, el $4 \%$ consideró casi siempre, el $28 \%$ a veces, el $64 \%$ casi nunca y el $4 \%$ nunca, mientras en el grupo experimental el $8 \%$ consideró siempre, el $68 \%$ casi siempre, el $20 \%$ a veces y el $4 \%$ casi nunca; estos resultado evidencia la mejora en el grupo experimental respecto a la dimensión apertura a los cambios tecnológicos en el colegio García Moreno del cantón El Guabo después de realizarse la intervención del programa de supervisión educativa. 
Resultados descriptivos de los recursos humanos

Tabla 5 Antes de la aplicación del programa

\begin{tabular}{|l|c|c|c|c|}
\hline & \multicolumn{2}{|c|}{$\begin{array}{c}\text { Grupo } \\
\text { control }\end{array}$} & \multicolumn{2}{c|}{$\begin{array}{c}\text { Grupo } \\
\text { experimental }\end{array}$} \\
\hline & $\mathrm{f}$ & $\%$ & $\mathrm{f}$ & $\%$ \\
\hline Siempre & 0 & $0 \%$ & 0 & $0 \%$ \\
\hline Casi siempre & 0 & $0 \%$ & 0 & $0 \%$ \\
\hline A veces & 11 & $44 \%$ & 7 & $28 \%$ \\
\hline Casi nunca & 12 & $48 \%$ & 13 & $52 \%$ \\
\hline Nunca & 2 & $8 \%$ & 5 & $20 \%$ \\
\hline
\end{tabular}

Fuente: Base de datos

Tabla 6: Después de la aplicación del programa

\begin{tabular}{|l|c|c|c|c|}
\hline & \multicolumn{2}{|c|}{$\begin{array}{c}\text { Grupo } \\
\text { control }\end{array}$} & \multicolumn{2}{c|}{$\begin{array}{c}\text { Grupo } \\
\text { experimental }\end{array}$} \\
\hline & $\mathrm{f}$ & $\%$ & $\mathrm{f}$ & $\%$ \\
\hline Siempre & 0 & $0 \%$ & 0 & $0 \%$ \\
\hline Casi siempre & 1 & $4 \%$ & 18 & $72 \%$ \\
\hline A veces & 8 & $32 \%$ & 7 & $28 \%$ \\
\hline Casi nunca & 14 & $56 \%$ & 0 & $0 \%$ \\
\hline Nunca & 2 & $8 \%$ & 0 & $0 \%$ \\
\hline
\end{tabular}

Fuente: Base de datos

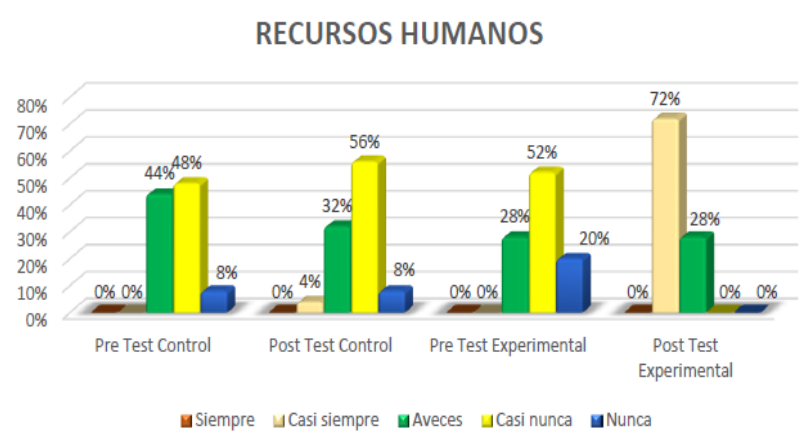

Figura 3. Niveles de la dimensión recursos humanos

En las tablas 5; 6 y en la figura 3 se observa que en el pre test con respecto al grupo control el $44 \%$ consideró a veces, el $48 \%$ casi nunca y el $8 \%$ nunca; mientras que en el grupo experimental el $28 \%$ a veces, el $52 \%$ consideró casi nunca, y el $20 \%$ nunca. En el pos test se observa que en el grupo control, el $4 \%$ consideró casi siempre, el 32\% a veces, el 56\% casi nunca y el $8 \%$ nunca, mientras en el grupo experimental el $72 \%$ consideró casi siempre, y el $28 \%$ a veces; este resultado evidencia la mejora en el grupo experimental respecto a la dimensión recursos humanos en el colegio García Moreno del cantón El Guabo después de realizarse la intervención del programa de supervisión educativa.

Resultados descriptivos de la comunicación.

Tabla 7 Antes de la aplicación del programa.

\begin{tabular}{|l|c|c|c|c|}
\hline & \multicolumn{2}{|c|}{$\begin{array}{c}\text { Grupo } \\
\text { control }\end{array}$} & \multicolumn{2}{c|}{$\begin{array}{c}\text { Grupo } \\
\text { experimental }\end{array}$} \\
\hline & $\mathrm{f}$ & $\%$ & $\mathrm{f}$ & $\%$ \\
\hline Siempre & 1 & $4 \%$ & 0 & $0 \%$ \\
\hline Casi siempre & 0 & $0 \%$ & 0 & $0 \%$ \\
\hline A veces & 5 & $20 \%$ & 7 & $28 \%$ \\
\hline Casi nunca & 15 & $60 \%$ & 11 & $44 \%$ \\
\hline Nunca & 4 & $16 \%$ & 7 & $28 \%$ \\
\hline
\end{tabular}

Fuente: Base de datos

Tabla 8 Después de la aplicación del programa

\begin{tabular}{|l|c|c|c|c|}
\hline & \multicolumn{2}{|c|}{$\begin{array}{c}\text { Grupo } \\
\text { control }\end{array}$} & \multicolumn{2}{c|}{$\begin{array}{c}\text { Grupo } \\
\text { experimental }\end{array}$} \\
\hline & $\mathrm{f}$ & $\%$ & $\mathrm{f}$ & $\%$ \\
\hline Siempre & 0 & $0 \%$ & 1 & $4 \%$ \\
\hline Casi siempre & 0 & $0 \%$ & 17 & $68 \%$ \\
\hline A veces & 8 & $32 \%$ & 7 & $28 \%$ \\
\hline Casi nunca & 15 & $60 \%$ & 0 & $0 \%$ \\
\hline Nunca & 2 & $8 \%$ & 0 & $0 \%$ \\
\hline
\end{tabular}

Fuente: Base de datos

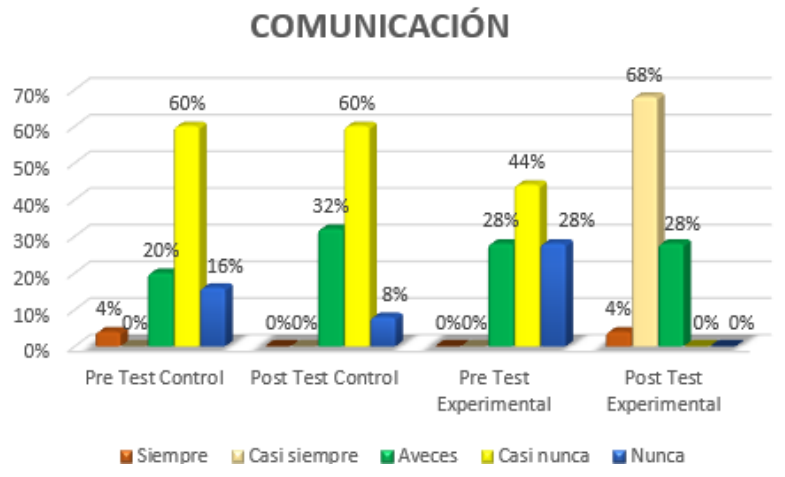

Figura 4. Niveles de la dimensión comunicación

En las tablas 7; 8 y en la figura 4 se observa que en el pre test con respecto al grupo control el $4 \%$ consideró siempre, el $20 \%$ consideró a veces, el $60 \%$ casi nunca y el $16 \%$ nunca; mientras que en el grupo experimental el $28 \%$ a veces, el $44 \%$ consideró casi nunca, y el $28 \%$ nunca. En el pos test se observa que en el grupo control, el 32\% a veces, el $60 \%$ casi nunca y el $8 \%$ nunca, mientras en el grupo experimental el $4 \%$ consideró siempre, el $68 \%$ consideró casi 
siempre, y el $28 \%$ a veces; este resultado evidencia la mejora en el grupo experimental respecto a la dimensión comunicación en el colegio García Moreno del cantón El Guabo después de realizarse la intervención del programa de supervisión educativa.

Resultados descriptivos de la motivación.

Tabla 9 Antes de la aplicación del programa

\begin{tabular}{|l|c|c|c|c|}
\hline & \multicolumn{2}{|c|}{$\begin{array}{c}\text { Grupo } \\
\text { control }\end{array}$} & \multicolumn{2}{c|}{$\begin{array}{c}\text { Grupo } \\
\text { experimental }\end{array}$} \\
\hline & f & $\%$ & f & $\%$ \\
\hline Siempre & 0 & $0 \%$ & 0 & $0 \%$ \\
\hline Casi siempre & 1 & $4 \%$ & 0 & $0 \%$ \\
\hline A veces & 8 & $32 \%$ & 9 & $36 \%$ \\
\hline Casi nunca & 15 & $60 \%$ & 14 & $56 \%$ \\
\hline Nunca & 1 & $4 \%$ & 2 & $8 \%$ \\
\hline
\end{tabular}

Fuente: Base de datos

Tabla 10 Después de la aplicación del programa

\begin{tabular}{|l|c|c|c|c|}
\hline & \multicolumn{2}{|c|}{$\begin{array}{c}\text { Grupo } \\
\text { control }\end{array}$} & \multicolumn{2}{c|}{$\begin{array}{c}\text { Grupo } \\
\text { experimental }\end{array}$} \\
\hline & $\mathrm{f}$ & $\%$ & $\mathrm{f}$ & $\%$ \\
\hline Siempre & 0 & $0 \%$ & 1 & $4 \%$ \\
\hline Casi siempre & 2 & $8 \%$ & 13 & $52 \%$ \\
\hline A veces & 6 & $24 \%$ & 9 & $36 \%$ \\
\hline Casi nunca & 13 & $52 \%$ & 1 & $4 \%$ \\
\hline Nunca & 4 & $16 \%$ & 1 & $4 \%$ \\
\hline
\end{tabular}

Fuente: Base de datos

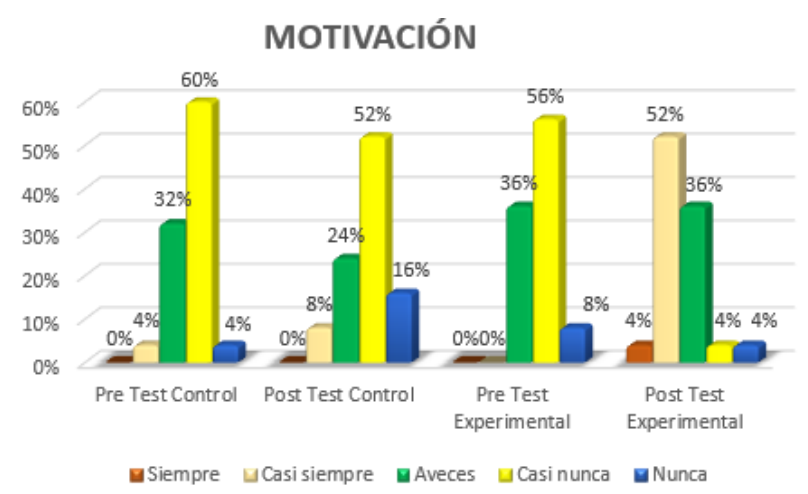

Figura 5. Niveles de la dimensión motivación

En las tablas 9; 10 y en la figura 5 se observa que en el pre test con respecto al grupo control el $4 \%$ consideró casi siempre, el $32 \%$ consideró a veces, el $60 \%$ casi nunca y el $4 \%$ nunca; mientras que en el grupo experimental el $36 \%$ a veces, el $56 \%$ consideró casi nunca, y el $8 \%$ nunca. En el pos test se observa que en el grupo control, el $8 \%$ casi siempre, el $24 \%$ a veces, el $52 \%$ casi nunca y el $16 \%$ nunca, mientras en el grupo experimental el $4 \%$ consideró siempre, el $52 \%$ consideró casi siempre, el $36 \%$ a veces, el $4 \%$ casi nunca y el $4 \%$ nunca; estos resultados evidencia la mejora en el grupo experimental respecto a la dimensión motivación en el colegio García Moreno del cantón El Guabo después de realizarse la intervención del programa de supervisión educativa.

Promedios por dimensiones del grupo control

Tabla 11 Promedios por dimensiones del grupo control

\begin{tabular}{|c|c|c|}
\hline & Pretest & Postest \\
\hline D1 & 12.6 & 12.72 \\
\hline D2 & 13.04 & 12.96 \\
\hline D3 & 12.08 & 12.36 \\
\hline D4 & 12.8 & 12.5 \\
\hline
\end{tabular}

Fuente: Base de datos

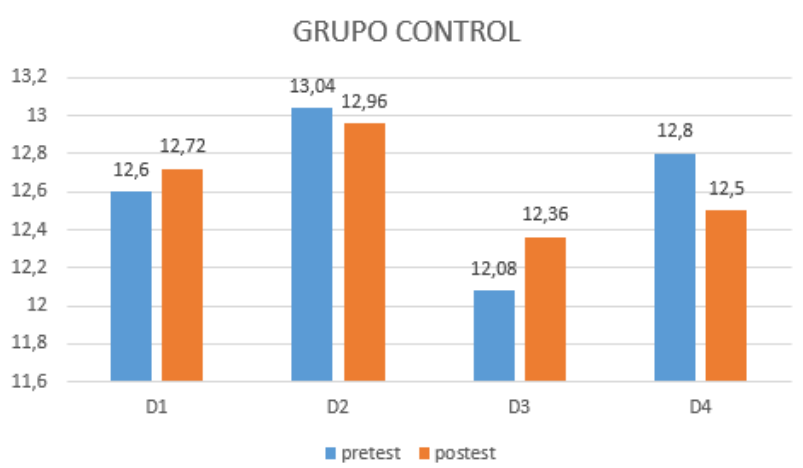

Figura 6. Promedios por dimensión del grupo control

En la tabla 11 y en la figura 6 se observa que en cuanto al grupo control en el pretest la dimensión recursos humanos es la que obtuvo mayor promedio y la más baja fue la dimensión apertura a los cambios tecnológicos mientras que en el postest la dimensión recursos humanos fue la que tuvo mayor promedio, pero la dimensión motivación fue la que tuvo menor promedio. 
Promedios por dimensiones del grupo experimental (motivación)

Tabla 12 Promedios por dimensiones del grupo experimental

\begin{tabular}{|c|c|c|}
\hline & Pretest & Postest \\
\hline D1 & 12.44 & 18.68 \\
\hline D2 & 11.8 & 18.6 \\
\hline D3 & 11.8 & 18.44 \\
\hline D4 & 12.6 & 17.48 \\
\hline
\end{tabular}

Fuente: Base de datos

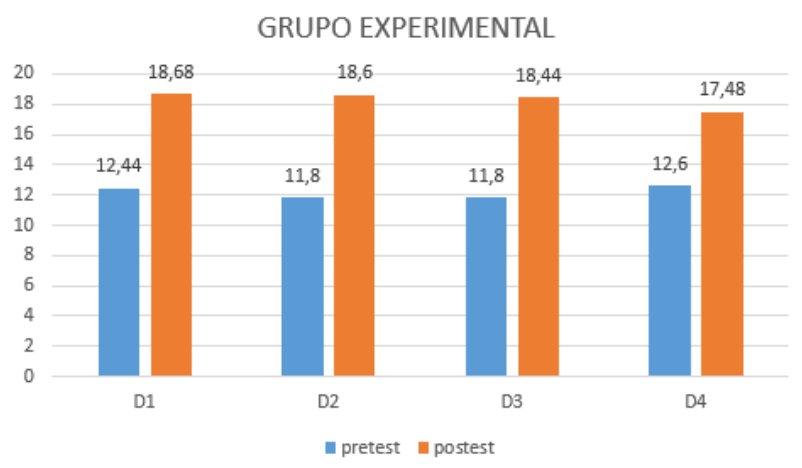

Figura 7. Promedios por dimensión del grupo experimental

En la tabla 12 y en la figura 7 se observa que en cuanto al grupo experimental en el pretest la dimensión motivación es la que obtuvo mayor promedio y la más baja fue la dimensión recursos humanos y comunicación; mientras que en el postest la dimensión apertura a los cambios tecnológicos fue la que tuvo mayor promedio, pero la dimensión motivación fue la que tuvo menor promedio.

\section{Discusión de los resultados}

Después de procesar los datos y analizar los resultados obtenidos se pudo afirmar que el clima organizacional mejora significativamente por la aplicación del programa de supervisión educativa en el colegio García Moreno del cantón El Guabo en Ecuador. Al respecto Mendoza (2005) afirma en su tesis la influencia de un programa de capacitación en liderazgo transformacional en el clima institucional, que la aplicación del programa de capacitación en liderazgo transformacional influye significativamente elevando el nivel de clima institucional.

Para Bezanque el cual evidenció en su estudio la dificultad de administrar una empresa lo cual afecta directamente al clima laboral por lo cual plantea la necesidad de un plan de mejora del clima organizacional, los cuales estuvo basados en conceptos novedosos, por ello sugiere que es necesario profundizar en el estudio del clima organizacional como clave para mejorar las actitudes de las personas que son parte de la institución, en el cual se considere factores como la buena relación entre compañeros, el interés de los directivos por su personal, lograr que las personas se sientan motivadas por el trabajo que realizan.

Gaibor (2013) en su estudio el autor llegó a precisar el rol importante que juega el clima organizacional para lograr una mayor eficacia en las organizaciones por lo cual considera que es necesario aplicar un plan de estrategias para que ello mejore, a lo que Gonzáles (2013) afirmó que todo ello optimiza la calidad de servicio que podemos brindar como institución.

En cuanto a la hipótesis específica primera se pudo afirmar que la aplicación del programa de supervisión educativa mejora significativamente la apertura a los cambios en el colegio García Moreno del cantón El Guabo en el Ecuador, ya que en el poste se obtuvo el valor de $\mathrm{p}=0,000<0,05$.

Al respecto Bustos (2001) agrega, que un buen clima o un mal clima organizacional, tendrá consecuencias para la organización a nivel positivo y negativo, definidas por la percepción que los miembros tienen de la organización.

Entre las consecuencias positivas, podemos nombrar las siguientes: logro, afiliación, poder, productividad, baja rotación, satisfacción, adaptación, innovación, etc. Entre las 
consecuencias negativas, podemos señalar: inadaptación, alta rotación, ausentismo, poca innovación, baja productividad, entre otras.

Respecto a la segunda hipótesis específica se pudo concluir que los recursos humanos mejoran significativamente por la aplicación del Programa de Supervisión educativa en el en el colegio García Moreno del cantón El Guabo en Ecuador; pues se obtuvo en el postest el valor de $p=0,000<0,05$.

Por su parte, Lepeley (2001:105) define el clima organizacional como "El ambiente social de la institución”. Bustos (2001: 7), coincide con los autores anteriores y sostiene que el clima, es la cualidad o propiedad del ambiente organizacional, que perciben o experimentan los miembros de la organización, y que influyen en su comportamiento. Para que una persona pueda trabajar bien debe sentirse bien consigo mismo y con todo lo que gira alrededor de ella y entender el ambiente donde se desenvuelve todo el personal.

Así mismo resultados similares obtuvo la investigación realizada por Chigne (2006), el cual señalo que existe relación entre el liderazgo, relaciones interpersonales y el clima organizacional afirma que existe una relación de dependencia positiva media entre el liderazgo y el clima organizacional; por ello se debe promover el liderazgo del directivo, el cual debe preocuparse por el recurso humano que forman sus docentes dentro de la institución; también Mendoza (2005) afirmó también que el aspecto relacionado a las recursos humanos como un factor importante en cuanto al clima organizacional.

Referente a la hipótesis específica tercera se pudo concluir que el plan de supervisión educativa mejora significativamente la comunicación en el colegio García Moreno del cantón El Guabo en Ecuador; al obtenerse en el postest que el valor de $\mathrm{p}=0,000<0,05$. Para la investigación el Clima Organizacional es el ambiente social que perciben los miembros de una institución, el cual es determinante en la forma que toma una organización, en las decisiones que al interior de ella se ejecutan o en cómo se tornan las relaciones dentro y fuera de la organización, por lo cual la comunicación es un aspecto importante que considerar

Finalmente la curta hipótesis específica se pudo evidenciar que la aplicación del plan de supervisión educativa mejora significativamente la motivación en el colegio García Moreno del cantón El Guabo en Ecuador; al obtenerse en el postest que el valor de $\mathrm{p}=0,000<0,05$; al respecto la investigación realizada por Tantalean (2002) en la cual el autor el autor precisa el trabajar la autoestima como una fuente de motivación para lograr un trabajo; por ello el plan de supervisión trabajó este aspecto para mejorar el nivel de motivación de los docentes; así también el estudio planteado por Morillo (2003) en la cual el autor implementó un plan de supervisión el cual generó mejoras en el desempeño del docente en aspectos como motivacionales, responsabilidades e identificación con la institución.

\section{Conclusiones}

De acuerdo con la hipótesis general planteada, se pudo determinar que el clima organizacional mejora significativamente por la aplicación del programa de supervisión educativa en el colegio García Moreno del cantón El Guabo en Ecuador, al obtenerse en el postest que el valor de $\mathrm{p}=, 000<0,05$, pudiéndose evidenciar diferencias significativas entre el pretest $\mathrm{y}$ postest gracias a la aplicación del programa de supervisión. 
En cuanto a la primera hipótesis específica, se pudo determinar que la aplicación del programa de supervisión educativa mejora significativamente la apertura a los cambios tecnológicos en el colegio García Moreno del cantón El Guabo en el Ecuador, ya que en el postest se obtuvo el valor de $\mathrm{p}=, 000<0,05$, pudiéndose evidenciar diferencias significativas entre el pretest y postest

Respecto a la segunda hipótesis específica, se pudo determinar que los recursos humanos mejoran significativamente por la aplicación del Programa de Supervisión educativa en el en el colegio García Moreno del cantón El Guabo en Ecuador; pues se obtuvo en el postest el valor de $\mathrm{p}=, 000<0,05$ pudiéndose evidenciar diferencias significativas entre el pretest $\mathrm{y}$ postest

Referente a la tercera hipótesis específica, se pudo concluir que el plan de supervisión educativa mejora significativamente la comunicación en el colegio García Moreno del cantón El Guabo en Ecuador; al obtenerse en el postest que el valor de $\mathrm{p}=, 000<0,05$ pudiéndose evidenciar diferencias significativas entre el pretest y postest

Respecto a la cuarta hipótesis específica se pudo concluir que el plan de supervisión educativa mejora significativamente la motivación en el colegio García Moreno del cantón El Guabo en Ecuador; al obtenerse en el postest que el valor de $\mathrm{p}=, 000<0,05$ pudiéndose evidenciar diferencias significativas entre el pretest $\mathrm{y}$ postest.

\section{Referencias Bibliográficas}

Aguilar, V. (2000). Paradigmas de la administración educativa. Lima: Ediciones JC.

Aguilar, V. (2004). Administración y supervisión educativa. Lima: Editorial Edigraber.

Alles, Martha. (2007). Comportamiento Organizacional. Argentina: Granica.

Alvarado Oyarce, Otoniel. (2001). Supervisión educativa orientada al control de calidad. Perú: Derrama Magisterial.

Alvarado Oyarce, Otoniel. (2002). Gestión Educativa: Enfoques y procesos. Perú: Universidad de Lima.

Alvarado Oyarce, Otoniel. (2005). Gestión de proyectos educativos. Perú: UNMSM.

Alvarado Oyarce, Otoniel. (2005). Gestión de proyectos educativos: lineamientos metodológicos. Perú: Universidad de San Marcos.

Anaya L y Paredes Rivera, J (2015) Propuesta de mejora de clima organizacional a partir de la gestión del talento humano (Tesis doctoral) Universidad del Pacifico

Arteaga V (2006) El grado de relación entre el liderazgo, relaciones interpersonales y el clima organizacional percibido por los trabajadores de la Institución Educativa Nacional "Víctor Andrés Belaúnde" de Trujillo

Tantaleán C, (2002) Clima organizacional de la Gran Unidad Escolar "José Faustino Sánchez Carrión" de Trujillo con el nivel de autoestima de los alumnos de segundo grado de secundaria en el año, (Tesis de maestría) Universidad Nacional de Trujillo

Chiavenato, Idalberto. (2004). Comportamiento Organizacional: la dinámica del éxito en las organizaciones. España: Edamsa.

Chiavenato, Idalberto. (2000). Introducción a la teoría general de la administración. México: Campus.

Espin J y Torres, N (2014) Análisis del clima organizacional y su impacto en el desempeño laboral del personal de aduanas del Ecuador, (Tesis de grado) Universidad Politécnica Salesiana 
Gaibor, María (2013) en su estudio titulado Análisis e implementación de estrategias para mejorar el clima laboral de la Unidad Educativa FAE $\mathrm{N}^{\circ} 3$

García, Bexabet (2015). "La supervisión educativa ¿víctima o excluida de las políticas públicas".UPEL. Año 2016, Vol 12, Nº1. Universidad Pedagógica Experimental Libertador. Maracay - Venezuela, pp 118 139.

García, María e Ibarra, Luis (2012). Características del clima organizacional. Eumed.net

Gonzales, Cindy y Soria, Vladimir (2013) Implementación de un sistema de mejoramiento del clima organizacional para mejorar la calidad del servicio que se brinde al usuario (Tesis de grado) Universidad Estatal Península de Santa Elena
Gómez Cueva, Romel. (2000). Administración de los Recursos Humanos en Instituciones Educativas. México: Trillas.

Hernández Sampieri, Roberto; Fernández Collado, Carlos y Baptista Lucio, Pilar. (2006). Metodología de la Investigación. México: Mc-GRAW-HILL.

Huaranga Ross, Oscar. (2000). Calidad educativa y enfoques constructivistas. Perú: San Marcos.

Lepeley, María Teresa. (2001). Gestión y Calidad en Educación: un modelo de evaluación. Chile: Salesianos.

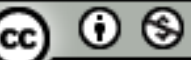

EY No Esta obra está bajo una licencia de Creative Commons Reconocimiento-No Comercial 4.0 Internacional. Copyright (c) Richard Hugo Clavijo Flores 